\title{
On linear instability mechanisms in incompressible open cavity flow
}

\author{
F. Meseguer-Garrido $\dagger$, J. de Vicente, E. Valero and V. Theofilis \\ School of Aerospace Engineering, Universidad Politécnica de Madrid, Plaza Cardenal Cisneros 3, \\ E-28040 Madrid, Spain
}

\begin{abstract}
A theoretical study of linear global instability of incompressible flow over a rectangular spanwise-periodic open cavity in an unconfined domain is presented. Comparisons with the limited number of results available in the literature are shown. Subsequently, the parameter space is scanned in a systematic manner, varying Reynolds number, incoming boundary-layer thickness and length-to-depth aspect ratio. This permits documenting the neutral curves and leading eigenmode characteristics of this flow. Correlations constructed using the results obtained collapse all available theoretical data on the three-dimensional instabilities.
\end{abstract}

Key words: instability, parametric instability

\section{Introduction}

Flow instabilities in an open cavity have been investigated in the past from the point of view of understanding the basic physical mechanisms involved, but also motivated by the multitude of industrial applications in which this configuration arises, such as open roofs on automobiles, landing gear in aircraft or weapon bays. Theoretical and numerical expediency have limited most analyses of open cavity flows to strictly two spatial dimensions, or three-dimensional domains in which spanwise periodicity is assumed. Fully non-periodic three-dimensional (TriGlobal) analysis still has a prohibitive computational cost, especially for geometries outside the simplest ones, although the first efforts regarding the TriGlobal analysis of the open cavity were recently made (Gómez 2013).

The majority of early theoretical work on the open cavity has revolved around the two-dimensional flow/acoustic resonance producing self-sustained oscillations in the shear layer (Rockwell 1977; Rockwell \& Naudascher 1979). In compressible flow, small-amplitude oscillations in the shear layer impinging at the downstream edge of the cavity generate pressure disturbances that travel upstream and reinforce the shear-layer oscillations. This feedback mechanism results in vortex shedding at the leading edge, which locks onto a characteristic frequency that follows the semi-empirical formula described by Rossiter (1964). Subsequent works postulated that in the incompressible regime this feedback can be considered as instantaneous, and in the experimental work of Sarohia (1975) two peaks of frequency that match the prediction of the Rossiter mechanism were found. Later Gharib \& Roshko (1987)

$\dagger$ Email address for correspondence: fernando.meseguer@upm.es 


\section{F. Meseguer-Garrido, J. de Vicente, E. Valero and V. Theofilis}

observed in their work on axisymmetric cavities that, as the incoming boundary-layer thickness decreased in relation to the depth of the cavity, there was a substantial change in behaviour of the cavity oscillations, characterised by a large-scale shedding from the cavity leading edge; the authors termed this finding the wake mode. Similar transitions have been observed by Pereira \& Sousa (1995) in two-dimensional incompressible direct numerical simulations (DNS) with laminar incoming boundary layer. Sipp \& Lebedev (2007) studied the bifurcation of the shear-layer modes in an incompressible confined cavity of aspect ratio $L / D=1$ at supercritical flow conditions, using weakly nonlinear analysis. Following that work, Barbagallo, Sipp \& Schmid (2009) and Sipp (2012) investigated the closed- and open-loop control problem, respectively, at those same conditions.

In the compressible regime, Colonius, Rowley \& Theofilis (2001) showed the first two-dimensional amplitude function in a two-dimensional open cavity, demonstrating a Tollmien-Schlichting wave emanating on the downstream wall and being connected with instability inside the open cavity (see also supplemental Appendix 4 of Theofilis 2011). The work of Rowley, Colonius \& Basu (2002) provided further insight regarding the onset of shear-layer oscillations from a steady flow and their nonlinear interactions, developing a criterion to predict the onset of disturbances from the steady flow. Later, Theofilis \& Colonius $(2003,2004)$ revisited the open cavity and, applying the residuals algorithm (Theofilis 2000b; Gómez et al. 2012; Gómez, Gómez \& Theofilis 2014) recovered the same Tollmien-Schlichting eigenmode as well as an acoustic mode connecting hydrodynamic perturbations inside the cavity with pressure fluctuations having their origin at the downstream cavity corner. Recently, Yamouni, Sipp \& Jacquin (2013) have given a detailed description of the effect of compressibility on the interaction of the acoustic feedback and resonance using global instability analysis in a two-dimensional cavity at a particular set of flow conditions and one Reynolds number. The authors confirmed that the shear-layer modes correspond to the beginning of branches of those global modes whose frequencies evolve with Mach number as described by Rossiter's semi-empirical formula, as is supported by previous findings by Sarohia (1975).

The thorough study of the two-dimensional self-sustained oscillations led to the discovery of three-dimensional structures in the flow, with frequencies far smaller than the ones of the Rossiter modes. Several studies reported these lower frequencies, mainly as a modulation of the two-dimensional shear-layer modes (Rockwell \& Knisely 1980; Koseff \& Street 1984; Neary \& Stephanoff 1987). The hypothesis that these modes were the result of nonlinear interaction between Rossiter modes was rebutted by Cattafesta et al. (1998) and Kegerise et al. (2004).

The aforementioned work by Theofilis \& Colonius (2004) also contained some of the first linear computations of a three-dimensional instability inside the cavity. The authors used a two-dimensional base flow multi-domain computation, and applying the appropriate boundary conditions to the upper side on the cavity subdomain they showed a structure consisting of a perturbation that coils around the main recirculating vortex. The first non-conforming spectral multi-domain approach for the numerical solution for the two-dimensional BiGlobal eigenvalue problem (EVP) was presented in de Vicente et al. (2004).

These three-dimensional perturbations were found to be dominant in some flow conditions by Brès \& Colonius (2008). This work is, to date, the most complete study on the compressible open cavity, and it established that the lower frequencies correspond to three-dimensional structures (independent from the shear-layer modes). These three-dimensional structures are sometimes known in the literature as centrifugal 
modes, and may qualitatively be related to instability modes seen in the lid-driven cavity (Theofilis 2000a; Albensoeder, Kuhlmann \& Rath 2001; González et al. 2011). Brès \& Colonius (2008) also confirmed the previously observed modulation of the two-dimensional shear-layer modes by the lower frequencies of the centrifugal modes.

There also exist in the literature numerous works on flow visualisation and characterisation of these three-dimensional open cavity instabilities. Recently, Faure et al. (2009) aimed to understand the three-dimensional flow morphology by varying the Reynolds number and the cavity shape. Basley et al. (2011) used time-resolved particle image velocimetry (PIV) to extract the spatial distribution of the most characteristic frequencies in the incompressible open cavity with two different aspect ratios, and also identified once again the presence of the aforementioned shear-layer modes in the incompressible regime. Alizard, Robinet \& Gloerfelt (2012) focused on the global instability analysis of open flows using a domain decomposition matrix-free method. One of the benchmark problems the latter authors employed is the square cavity, where, at a single Reynolds number, the three-dimensional spanwise-periodic leading perturbations were identified.

Despite this previous work a complete study on three-dimensional incompressible cavity flow is still missing. More recently, the work of de Vicente et al. (2014) presented a combined theoretical, numerical and experimental work on global instability of the open cavity in incompressible flow, which also includes validation and verification of the analysis results, the focus being on the three-dimensional structures, dominant in the studied flow regime. The aim of the present work is to present an in-depth extension of the theoretical results presented in that work, revisiting incompressible flow over the spanwise-periodic rectangular cavity. A systematic global (BiGlobal) instability analysis is performed, taking into account the effect of parameters that, no doubt on account of the cost of this type of analysis, have either been studied incompletely or been altogether ignored in the literature. The incoming boundary-layer thickness and the Reynolds number based on the dimensions of the cavity are varied independently, as is the spanwise wavenumber, for a range of length-to-depth aspect ratios, until the most interesting part of the spectrum containing all amplified global eigenmodes is revealed.

Section 2 describes the problem at hand and puts in context previous findings. Results of parametric analyses are shown in $\$ \S 3$ and 4 , where the critical Reynolds numbers and the leading perturbations at all conditions studied are identified. Two-dimensional instabilities concordant with the literature have been found and are described in $\$ 3$. A synthesis of three-dimensional global instability analysis results obtained herein provides analytical expressions for the relation between critical Reynolds number and both incoming boundary-layer thickness and cavity aspect ratio, which are given in $\S 4.3$. The connection between the characteristic frequency of the leading global eigenmodes and the cavity aspect ratio is revealed from our results.

\section{Problem description}

A schematic description of the configuration is depicted in figure 1 . The main geometrical parameters considered are the length-to-depth ratio $(L / D)$ and the wavelength in the spanwise direction $\left(L_{z}\right)$ with the corresponding wavenumber $\left(\beta=2 \pi / L_{z}\right)$. Regarding the flow features, Reynolds number based on cavity depth $\left(R e_{D}\right)$ and the incoming boundary-layer thickness $(\delta / D)$ have been chosen to characterise the flow. The subscript on $\delta$ denotes the position in the streamwise direction where the boundary-layer thickness is measured. So, $\delta_{0} / D$ corresponds to 


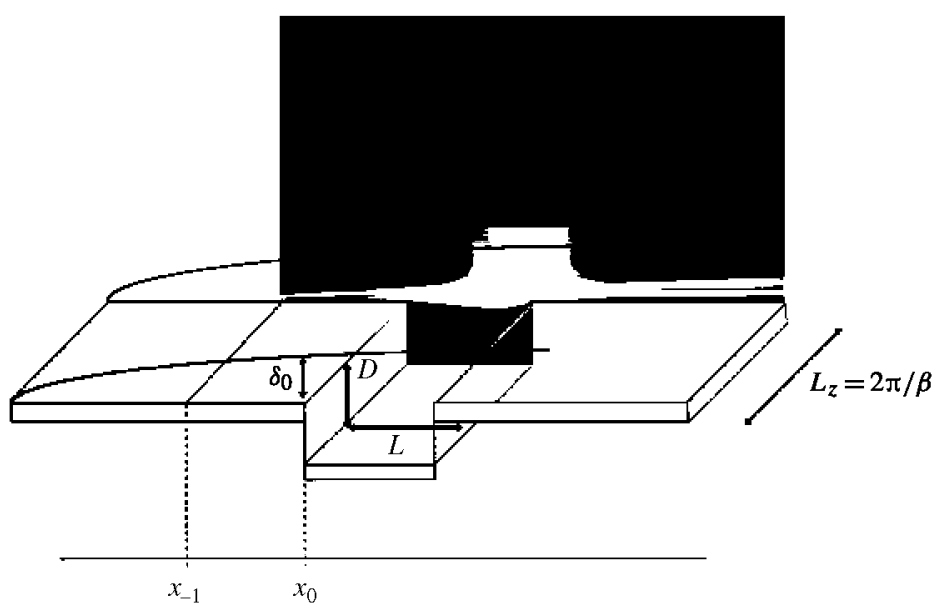

FIGURE 1. (Colour online) Schematic description of the three-dimensional open cavity and problem parameters.

the leading edge of the cavity while $\delta_{-1} / D$ is the beginning of the computational domain. The boundary-layer displacement thickness $(\theta / D)$ has been computed in order to compare with the literature.

\subsection{Base flow}

The steady two-dimensional basic flow required for the BiGlobal instability analysis of the open cavity flow is obtained using a semi-implicit artificial compressibility method.

Using this method, the Navier-Stokes equations governing the motion of a viscous incompressible fluid become:

$$
\begin{gathered}
\frac{\partial \boldsymbol{u}}{\partial t}+(\boldsymbol{u} \cdot \nabla) \boldsymbol{u}=\frac{1}{R e_{D}} \nabla^{2} \boldsymbol{u}-\nabla P, \\
\varepsilon \frac{\partial P}{\partial t}+\nabla \cdot \boldsymbol{u}=0,
\end{gathered}
$$

where $\boldsymbol{u}$ represents the velocity vector comprising the two velocity components in the streamwise and wall-normal directions $(u, v), P$ is the pressure and $\varepsilon$ is an arbitrarily small parameter for the artificial compressibility component. The Chebyshev spectral collocation technique has been chosen for the spatial discretisation, while time advance has been performed using a semi-implicit Euler scheme leading to the following system of discretised equations:

$$
\left.\begin{array}{l}
\frac{u^{n+1}-u^{n}}{\Delta t}-\frac{1}{R e_{D}} \nabla^{2} u^{n+1}+P_{x}^{n+1}=-\left(u^{n} u_{x}^{n}+v^{n} u_{y}^{n}\right), \\
\frac{v^{n+1}-v^{n}}{\Delta t}-\frac{1}{R e_{D}} \nabla^{2} v^{n+1}+P_{y}^{n+1}=-\left(u^{n} v_{x}^{n}+v^{n} v_{y}^{n}\right), \\
\varepsilon \frac{P^{n+1}-P^{n}}{\Delta t}-u_{x}^{n+1}-v_{y}^{n+1}=0 .
\end{array}\right\}
$$

This semi-implicit numerical scheme treats convection terms in the Navier-Stokes equation explicitly but pressure and viscous terms implicitly. This approach avoids 


\section{On linear instability mechanisms in incompressible open cavity flow}

solving a nonlinear problem at each time step while preserving the stability properties of implicit solvers. The choice of an Euler method for time advancing is due to its simple implementation and its good stability properties when a steady solution is sought.

The main drawback of this kind of method is the loss of accuracy before the steady state is reached. This is not an issue here, since in the BiGlobal analysis methodology a steady base solution is sought.

The spectral multi-domain methodology implemented is here fully exploited by decomposing the computational domain into rectangular subdomains. The presence of a laminar boundary layer upstream of the cavity, the shear layer developed from the leading edge, as well as the boundary layer on the downstream cavity wall all require a fine grid to be properly resolved. A stretching law permits the concentration of the Chebyshev collocation points in the most interesting part of the domain and consequently leads to a better resolution.

The computational domain must be defined so that it is large enough to isolate as much as possible the numerical effects of imposing boundary conditions for open flows.

In the open cavity flow four different kinds of boundaries appear: wall, inflow, far field and outflow. The first issue concerns the implementation of proper boundary conditions. For viscous wall-bounded flows no-slip boundary conditions are adequate for the velocity, while homogeneous Neumann conditions for the pressure derivative in the normal direction to the wall are applied.

The velocity components of the laminar boundary layer at the inflow of the computational domain are calculated by integrating the Blasius equation. Several Blasius profiles have been computed and used in order to obtain a range of basic flows at different Reynolds numbers and with different incoming boundary-layer thickness, as required by the subsequent instability analysis. In the part of the study in which the boundary-layer thickness was not modified it was fixed to a reference value at the start of the computational domain $\left(\delta_{-1}\right)$. This reference value for the incoming Blasius profile was chosen to be the same as in Rowley et al. (2002) and Brès \& Colonius (2008).

This condition, originally chosen to match the works of the aforementioned authors, is unconventional, in the sense that the parameter kept constant is not the one that is supposed to dominate the behaviour of the problem (which would be the boundary-layer thickness at the leading edge of the cavity), but a computational one (the incoming boundary-layer thickness at the start of the computational domain). That means that the constant incoming boundary-layer thickness results correspond in reality not to a plane of such constant thickness, but to a manifold in the parameter space, as it was described before. The general effect on the results of this is, nevertheless, small, and in any case the effect of said incoming boundary-layer thickness has also been thoroughly studied, as will be seen in what follows.

So, the following boundary condition is defined at the inflow boundary:

$$
\begin{aligned}
& \bar{u}=\bar{u}_{\text {Blasius }}, \\
& \bar{v}=\bar{v}_{\text {Blasius }},
\end{aligned}
$$

while, as in standard boundary-layer flows, Dirichlet conditions for the velocity field lead to homogeneous Neumann conditions for pressure in the normal direction. The far-field boundary denotes the upper artificial limit, far enough from the boundary layer, where the streamwise component of the velocity vector is equal to a characteristic velocity. Normal velocity is considered constant in the $y$ direction. 


\section{F. Meseguer-Garrido, J. de Vicente, E. Valero and V. Theofilis}

The choice and implementation of the boundary condition in the downstream limit of the computational domain has been the most critical issue in the convergence of the steady basic flow calculation. The boundary condition which has shown the best performance consists of forcing the second derivatives of the flux velocity components to vanish in the outflow while keeping the pressure constant with a Dirichlet boundary condition (Gresho \& Sani 1987; Sani \& Gresho 1994):

$$
\frac{\partial^{2} \bar{u}}{\partial x^{2}}=0, \quad \frac{\partial^{2} \bar{v}}{\partial x^{2}}=0, \quad \bar{P}=\bar{P}_{0} .
$$

\subsection{BiGlobal instability analysis}

Linear stability analysis in the BiGlobal framework involves the substitution of a decomposition of any of the independent flow variables, e.g. the three velocity components, temperature and pressure $q(x, y, z, t)=(u, v, w, T, P)^{\mathrm{T}}$, into the (incompressible or compressible) equations of motion. All quantities are considered to be composed of an $O(1)$ steady two-dimensional basic state and small-amplitude $O(\epsilon)$ unsteady three-dimensional perturbations, according to the BiGlobal ansatz,

$$
\boldsymbol{q}(x, y, z, t)=\overline{\boldsymbol{q}}(x, y)+\epsilon \hat{\boldsymbol{q}}(x, y) \mathrm{e}^{\mathrm{i}(\beta z-\omega t)},
$$

for the determination of the complex eigenvalue:

$$
\omega=2 \pi \frac{S t_{D} U_{\infty}}{D}+\mathrm{i} \sigma
$$

In the temporal framework used, the Strouhal number $(S t)$ represents a dimensionless frequency and $\sigma$ is the amplification/damping rate of the disturbance sought, while barred and hatted quantities denote basic and disturbance flow quantities, respectively. Spatial homogeneity along the direction $z$ is assumed, in which the wavenumber parameter $\beta$ is introduced, related to a periodicity length $L_{z}$ along this direction by $L_{z}=2 \pi / \beta$.

Substituting the decomposition (2.7) in the linearised Navier-Stokes equations results in the following generalised real non-symmetric partial-derivative EVP:

$$
\begin{gathered}
{\left[\mathscr{L}_{\varepsilon}-\left(\mathscr{D}_{x} \bar{u}\right)\right] \hat{u}-\left(\mathscr{D}_{y} \bar{u}\right) \hat{v}-\mathscr{D}_{x} \hat{P}=\omega \hat{u}} \\
-\left(\mathscr{D}_{x} \bar{v}\right) \hat{u}+\left[\mathscr{L}_{\varepsilon}-\left(\mathscr{D}_{y} \bar{v}\right)\right] \hat{v}-\mathscr{D}_{y} \hat{P}=\omega \hat{v} \\
\mathscr{L}_{\varepsilon} \hat{w}-\beta \hat{P}=\omega \hat{w} \\
\mathscr{D}_{x} \hat{u}+\mathscr{D}_{y} \hat{v}-\beta \hat{w}=0
\end{gathered}
$$

where

$$
\mathscr{L}_{\varepsilon}=\frac{1}{R e_{D}}\left(\frac{\partial^{2}}{\partial x^{2}}+\frac{\partial^{2}}{\partial y^{2}}-\beta^{2}\right)-\bar{u} \frac{\partial}{\partial x}-\bar{v} \frac{\partial}{\partial y},
$$

and $\mathscr{D}_{x}, \mathscr{D}_{y}$ are the corresponding numerical differentiation operators in the $x$ and $y$ directions respectively.

The problem is completed with the imposition of boundary conditions for the disturbance quantities. At solid walls no-slip boundary conditions $\hat{u}=\hat{v}=\hat{w}=0$ are imposed for the velocity components while the homogeneous Neumann condition has been found to be the best for the disturbance pressure. Boundary conditions for the 
On linear instability mechanisms in incompressible open cavity flow

disturbance pressure do not exist physically, but compatibility is ensured with the choice of normal first derivative equal to zero:

$$
\frac{\partial \hat{P}}{\partial n}=0 .
$$

The inflow boundary is treated in the same way, considering there are no velocity fluctuations across this boundary; homogeneous Neumann boundary conditions are imposed there for the pressure perturbations. At the outflow boundary Neumann boundary conditions are applied on all the perturbation variables:

$$
\frac{\partial \hat{u}}{\partial n}=\frac{\partial \hat{v}}{\partial n}=\frac{\partial \hat{w}}{\partial n}=\frac{\partial \hat{P}}{\partial n}=0 .
$$

The far field, the top boundary of the computational domain, has been initially treated like the inflow condition. This choice has been proved to be correct for the leading cavity modes, whose structure is confined within the cavity. On the other hand, these conditions strongly affect the growth rate of the two-dimensional shear-layer modes.

For a detailed discussion on the boundary conditions used in this analysis the interested reader is referred to de Vicente (2010). The spatial domain is decomposed using the same multi-domain methodology as explained in the base flow section, each domain being discretised using the Chebyshev collocation technique.

The resulting two-dimensional BiGlobal EVP:

$$
A x=\omega M x,
$$

is solved by Krylov subspace iteration in two stages. First, the matrix discretising the EVP is LU-decomposed (though never formed explicitly); second, the decomposition is fed into an Arnoldi iteration with shift-and-invert methodology to recover the leading eigenvalues closest to the stability bound.

\section{The two-dimensional limit}

The two-dimensional flow over a rectangular cavity has been widely analysed in the literature. For the sake of completeness, a two-dimensional BiGlobal $(\beta \rightarrow 0)$ analysis of the stability of the laminar two-dimensional incompressible cavity flow is first discussed.

In figure 2 the characteristic frequency of the least stable two-dimensional eigenmodes in the range of $R e_{D}$ and $\delta_{0} / D$ studied (shown in table 1 ) is presented for $L / D=2$ and $L / D=3$. As can be seen, the frequency of the two shear-layer modes found in this analysis matches the range predicted in the literature (Sarohia 1975; Rowley et al. 2002; Brès 2007; Basley et al. 2013; Yamouni et al. 2013). The reduction in the Strouhal number of the modes with the increase of the incoming boundary-layer thickness reported here was already observed in Yamouni et al. (2013). Regarding the amplification of those modes, the behaviour is consistent with previous results in the literature: when the shear layer becomes thicker the Kelvin-Helmholtz instability damps until the layer becomes stable, as has been observed experimentally (Gharib \& Roshko 1987; Rockwell \& Knisely 1987). In figure 3 the velocity profiles of the reconstruction of the total flow consisting of the base flow plus the first of those modes is shown. The shedding of vortices due to the impingement of the shear layer on the trailing edge can be seen. 


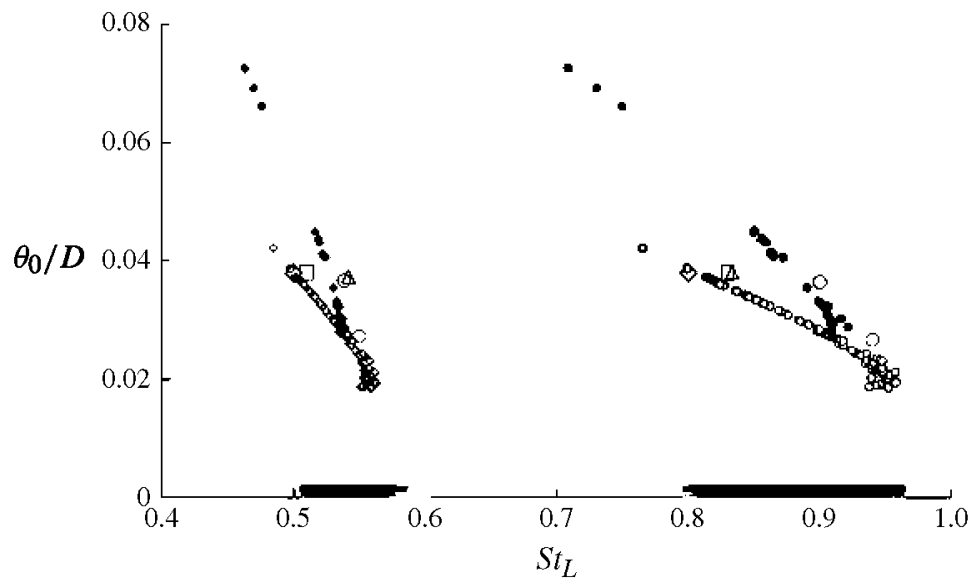

FIGURE 2. (Colour online) Variation of the dimensionless frequency (Strouhal number based on cavity length $S t_{L}$ ) of the two least stable eigenmodes (small circles and diamonds respectively) in the two-dimensional limit with the incoming boundary-layer thickness: $L / D=2$ open symbols, and $L / D=3$ filled symbols. The grey strips show the range of Strouhal numbers obtained by Sarohia (1975), and the larger (red online) open symbols the results of several studies at $L / D=2$ : squares, the $2 \mathrm{M} 2$ run of Rowley et al. (2002); diamonds, the Brès (2007) $M=0.3$ run; triangles, the lower runs of Yamouni et al. (2013); circles, points from Basley et al. (2013).

$$
\begin{aligned}
& L / D \quad 1.0,1.1,1.2,1.3,1.4,1.5,2 \text { and } 3 \\
& \operatorname{Re}_{D} \quad \sim 800-4600 \\
& \beta \quad \sim 0-22 \\
& \delta_{0} / D \quad \sim 0.05-0.3
\end{aligned}
$$

TABLE 1. Range of the parameters studied.
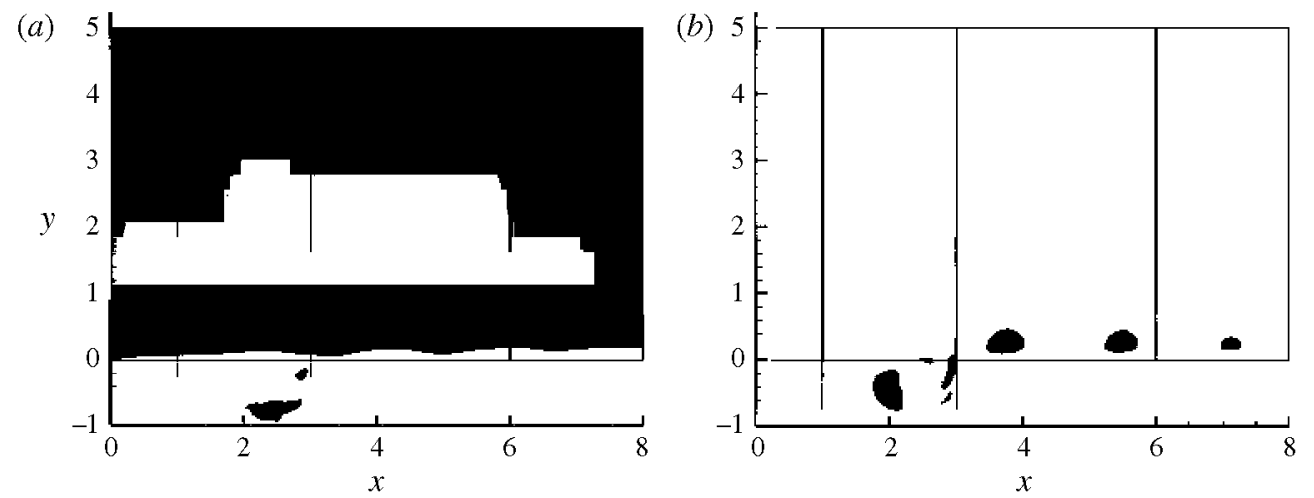

FIGURE 3. (Colour online) Velocity profiles of the reconstructed two-dimensional flow with the first shear-layer mode: $(a)$ streamwise velocity, and (b) wall-normal velocity. 


\section{On linear instability mechanisms in incompressible open cavity flow}

\section{Three-dimensional global instability analysis}

The parametric dependence of the stability features of the flow is now discussed in a three-dimensional framework $(\beta \neq 0)$. Besides the geometric parameter of aspect ratio $L / D$, the wavelength in the spanwise direction, $\beta$, is also examined. In addition, the effect of the incoming boundary-layer thickness and the Reynolds number has been investigated in this section. Table 1 contains the ranges of the different parameters examined.

The separation between the stable and unstable regimes in the parametric space is a hypersurface. This manifold marks the change of stability of the critical eigenvalue at each point of the parameter space. Owing to its multi-dimensional nature, the representation of that hypersurface is rather complex, so in order to characterise the stability boundaries neutral curves are presented as cuts through the hypersurface with parameter planes $R e_{D}$ vs. $\beta$, or $R e_{D}$ vs. $\theta_{0} / D$. In the next subsections, first the effect of Reynolds number and spanwise wavelength on the stability features of the eigenmodes is presented. Subsequently the consequence of varying the incoming boundary-layer thickness $\theta_{0} / D$ and aspect ratio $L / D$ is analysed. To conclude this section a synthesis of the results including the relevant parametric dependences is described.

\subsection{Reynolds number and $\beta$}

The spanwise wavenumber, $\beta$, characterises the instability modes rather than being a parameter of the problem, but it is nonetheless a parameter of the analysis. In an idealised spanwise-infinite cavity the first structures to amplify in a linear context would be the ones that correspond to the most unstable for that given point in the parameter space. When dealing with three-dimensional centrifugal instabilities two characteristic spanwise lengths for the periodic instabilities are found to be inherently more unstable, namely, perturbations that are of the size of the cavity depth, $\beta \simeq 6$, and also perturbations with a spanwise length of about one half of the cavity depth, $\beta \simeq 12$. In addition, the two-dimensional instabilities, previously seen in $\S 3$, also tend to appear as a third main inherently unstable area in the parameter space. The effect of Reynolds number on the flow stability has also been studied. In general, as $R e_{D}$ increases so does the amplification/damping rate (denoted $\sigma$ ) for all the eigenvalues, as increasing $R e_{D}$ means increasing the velocity gradient at the shear layer, which is one of the main mechanisms for introducing energy into the small perturbations.

In figure 4 the neutral curves for $L / D=2$ are shown. There are three main unstable modes. The first one to become unstable (Mode I) for this configuration, shown in grey circles in the figure, is the mode associated with a pair of complex-conjugated eigenvalues, known in the literature as a travelling mode. The second mode, open circles, undergoes a bifurcation with the variation of $\beta$. For low wavenumbers, there are two complex-conjugated eigenvalues (so it is also a travelling mode) but when $\beta$ grows those two eigenvalues collapse and become two real eigenvalues, one of which gets stabilised, and the other one destabilised. This disturbance will be named Mode II in what follows. The third unstable eigenmode shown, crosses, is also a travelling disturbance. That third mode is of little relevance and it is only present for relatively high- $R e_{D}$ configurations. In figure 5 a comparison of the amplification of the modes with the run of Brès \& Colonius (2008) that is closest to incompressible flow (2M01) is shown. Their results are superposed as open diamonds upon our results. The three most significant modes reported by them are found to correspond to conditions close to maximum amplification of the two modes found in the present work, while the fourth mode reported by Brès \& Colonius (2008) belongs to a different branch, albeit not at maximum amplification conditions. 




FiguRE 4. Neutral curves for $L / D=2$, and $\theta_{-1} / D=0.0337$ : $\bullet$, Mode I; $\mathrm{O}$, Mode II (bifurcated); $x$, third mode.

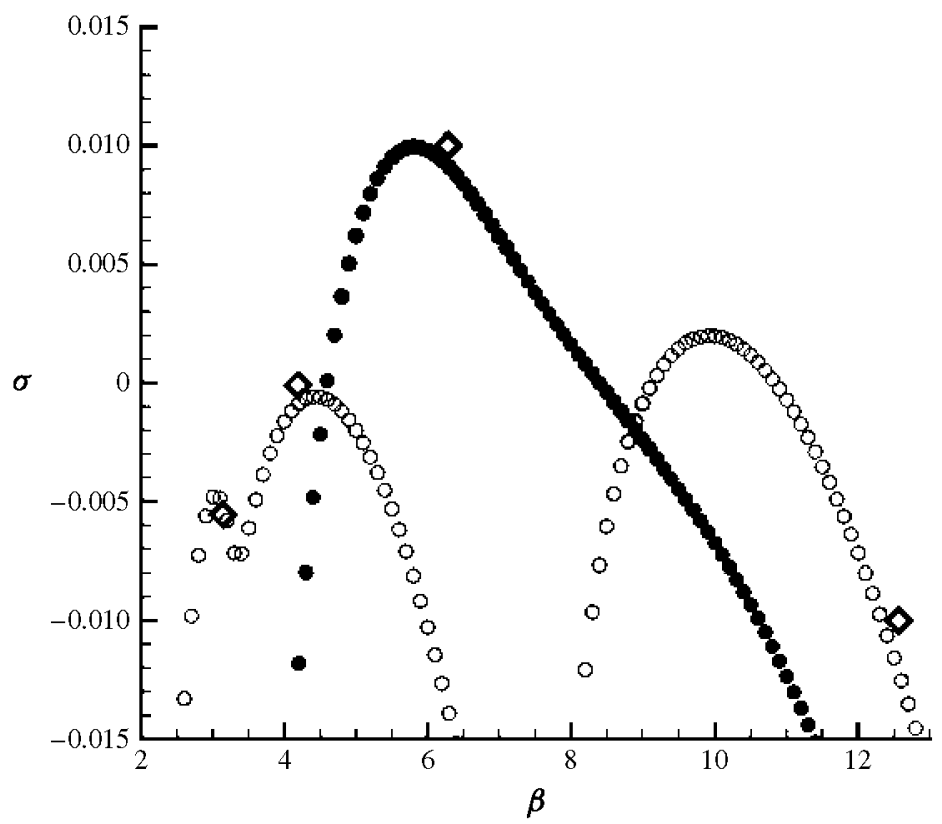

Figure 5. (Colour online) Amplification versus $\beta$ for $R e_{D}=1500$ : $\bullet$, Mode I; O, Mode II (bifurcated); and $\diamond$, results from Brès \& Colonius (2008). 
On linear instability mechanisms in incompressible open cavity flow

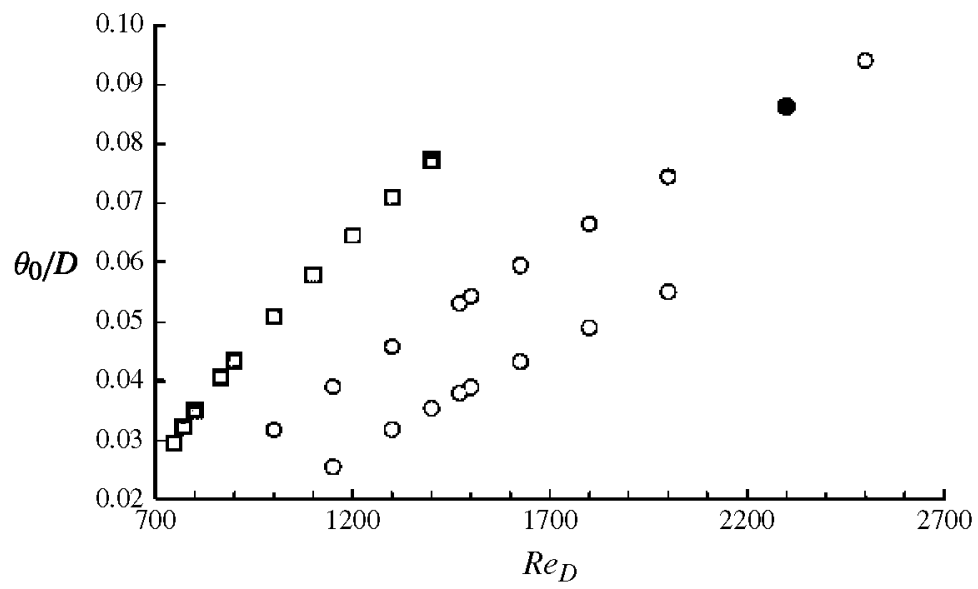

FIGURE 6. Neutral curves in the $R e_{D}$ vs. $\theta_{0} / D$ plane for the critical $\beta$ : filled symbols, Mode I ; open symbols, Mode II (bifurcated). $L / D=2$ is shown in circles $(\bullet, O)$ and $L / D=3$ in squares (ם).

\subsection{Incoming boundary-layer thickness $\theta_{0} / D$ and aspect ratio $L / D$}

In figure 6 the effect of incoming boundary-layer thickness $\theta_{0} / D$ on flow stability is shown. A parametric sweep in the $R e_{D}$ vs. $\theta_{0} / D$ space is presented for Mode I and Mode II for $L / D=2$, and Mode I for $L / D=3$. It is important to remark that these three curves do not correspond to constant- $\beta$ planes. Both increasing $R e_{D}$ and decreasing boundary-layer thickness leads to larger gradients, and eventually to destabilisation of the flow. The effect of decreasing the boundary-layer thickness is qualitatively similar to increasing $R e_{D}$ for the three-dimensional instabilities, while it has a much more pronounced effect on the two-dimensional shear-layer modes.

The effect of the aspect ratio on the stability is studied next and the relation between the size of the perturbations and the critical Reynolds number based on $D$ is documented (see $\S 4.3$ ). When $L \simeq D$ the size of the perturbation is constrained by the length of the cavity. Once $L / D$ is big enough the vortex over which the perturbations develop is not bounded by the cavity length. That means that the size of the perturbation, denoted $L_{P} / D$, is determined by the size of the recirculating vortex, not directly by $L / D$. In addition, the different behaviour of the two modes with $L / D$ leads to significant differences in the neutral curves for each aspect ratio, as can be seen in figure 7. The variation of the critical Reynolds number of the two different lobes (when present) of Modes I and II with $L / D$ for constant $\delta_{-1} / D$ is shown in figure $8(a)$.

Mode I presents a lobe with $\beta \sim 12$ for low $L / D$. In the case of $L / D=1$ this is the second mode to become unstable (figure $7 a$ ). When the aspect ratio increases up to $L / D=1.1$ a new lobe of that mode becomes unstable in the range of Reynolds numbers studied, with a maximum amplification on the range of $\beta \sim 6$. For the $L / D=$ 1.2 cavity there is a change in the leading instability of this mode, as the low- $\beta$ lobe is the first one to become unstable. Increasing the aspect ratio even more to $L / D=1.3$ leads to the main shift in stability, as Mode I becomes the first one to become unstable, while the second lobe of that mode, now on $\beta \sim 11$ starts to vanish. By $L / D=1.4$ the mode comprises a single lobe with a maximum in the range of $\beta \sim 6$, and a slight lump of increased instability around $\beta \sim 11$. As $L / D$ continues to 

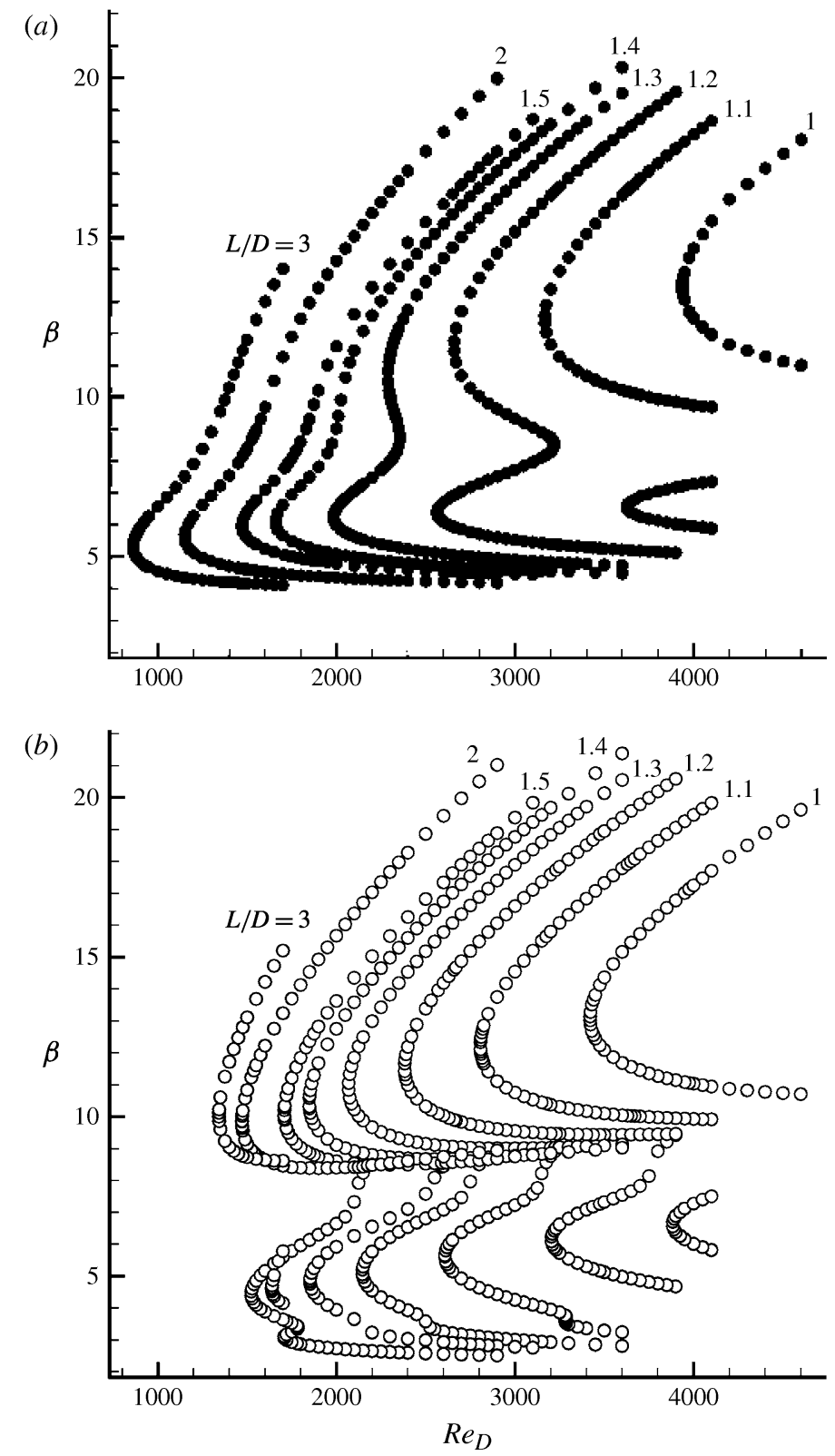

Figure 7. Neutral curves for $\theta_{-1} / D=0.0337$ : $(a)$ Mode I (@), (b) Mode II (O). Aspect ratio varying from $L / D=1$ (higher $R e_{D}$ ) to $L / D=3$ (lower $R e_{D}$ ) with the values listed in table 1.

increase, to 1.5, 2 and 3 the basic shape of the instability curves of the mode remains unmodified and only a change in the rate of variation of the critical $R e_{D}$ can be seen, as it will be discussed in the next section.

Mode II (figure $7 b$ ) has even more interesting behaviour, being a bifurcated mode. At lower $\beta$ a complex-conjugate pair of eigenvalues is found in the analysis. Around $\beta \sim 9$ a bifurcation occurs, and two real modes appear, one becoming more unstable 



FIGURE 8. Variation with $L / D$ and $L_{P} / D$ of $(a)$ the critical $\operatorname{Re}_{D}$ for the critical $\beta$ and $(b)$ the $\beta$ of maximum amplification. All data are for $\theta_{-1} / D=0.0337$. Mode I, filled symbols; Mode II, open symbols, with circles for low $\beta(O, \bullet)$ and diamonds high $\beta(\diamond, \diamond)$. Lines show the predicted curves of (4.1), and the hyperbolic law described in $\S 4.3$. Small symbols show the equivalent points for $L_{P} / D$.

( $\sigma$ increases), and the other one more stable, as $\sigma$ decreases. So for $L / D=1$ the most unstable eigenmode corresponds to the real branch of the bifurcated mode, with a maximum amplification around $\beta \sim 12$, i.e. a characteristic periodicity length around $D / 2$. In the case of this mode the most unstable part is always the real branch of high $\beta$. The difference in critical Reynolds number between the two branches decreases as $L / D$ increases. This mode is the critical one for $L / D=1.2$ and lower, as was discussed above when explaining the features of Mode I. At $L / D=1.3$ and 2 there is a small increase of instability in the zone of $\beta \sim 3$ and an small lobe is formed. In the case of $L / D=1.4$ and 1.5 there is a turning point in that region, but a complete lobe is not formed. That change of behaviour is interesting, because the trend is not continuous with the change of the $L / D$ parameter. Also, the only instance of critical $R e_{D}$ increasing as $L / D$ increases can be seen in the travelling branch of this mode, for $L / D=3$. 


\begin{tabular}{|c|c|c|c|c|c|c|c|c|c|c|}
\hline \multirow[b]{2}{*}{$L / D$} & \multicolumn{4}{|c|}{ Mode I } & \multicolumn{4}{|c|}{ Mode II } & \multicolumn{2}{|c|}{ Third mode } \\
\hline & $R e_{D}$ & $\beta$ & $R e_{D}$ & $\beta$ & $R e_{D}$ & $\beta$ & $R e_{D}$ & $\beta$ & $R e_{D}$ & $\beta$ \\
\hline 10 & - & - & 3937 & 13.47 & - & - & 3421 & 13.07 & - & - \\
\hline & 3630 & 6.56 & 3167 & 12.45 & 3884 & 6.63 & 2807 & 12.22 & - & - \\
\hline & 2565 & 6.38 & 2659 & 11.60 & 3205 & 6.16 & 2383 & 11.54 & 3476 & 11.61 \\
\hline & 1985 & 6.23 & 2289 & 10.66 & 2608 & 5.63 & 2069 & 10.95 & 3071 & 11.07 \\
\hline & 1659 & 6.09 & - & - & 2147 & 5.13 & 1849 & 10.45 & 2863 & 10.88 \\
\hline & 1472 & 5.96 & - & - & 1853 & 4.84 & 1708 & 10.11 & 2724 & 10.83 \\
\hline & 1150 & 5.62 & - & - & 1523 & 4.45 & 1471 & 9.86 & 2207 & 10.34 \\
\hline & 865 & 5.30 & 一 & - & - & - & 1342 & 10.06 & - & - \\
\hline
\end{tabular}

TABLE 2. Critical parameters of the first three modes for each of the aspect ratios and for constant $\delta_{-1} / D$.

A summary of the main characteristics of the critical values when a change in stability occurs for constant $\delta_{-1} / D$ is shown in table 2 . This table serves as a reference for the rest of the figures of the paper.

\subsection{Synthesis of the results}

In this section a description of relations between parameters of the flow and its global instability characteristics is presented. An empirical law is provided when possible.

\subsubsection{Incoming boundary-layer thickness and Reynolds number}

In figure 6 it can be appreciated that for sufficiently thick boundary layers there seems to exist a linear relation between the critical $R e_{D}$ and the incoming boundarylayer thickness for the modes studied. Mode I for $L / D=3$ presents a saturation regime for thin boundary layers in which that linear behaviour does not occur. The slope of the linear part of the curve depends on the length on which the Reynolds number is based. Basing $R e$ on $L$ instead of $D$ causes the neutral curves of Mode I for both $L / D=2$ and $L / D=3$ pictured in figure 6 to have the same slope in the higher Reynolds number range.

\subsubsection{Parametric dependence on aspect ratio}

The results of figure $8(a)$ indicate that an exponential fit can be constructed between the critical $R e_{D}$ of the modes and the inverse of the aspect ratio, that is, $D / L$, for aspect ratios lower than $L / D=1.5$. To explain that change in behaviour for higher aspect ratios, figure 9 contains a cut of the spanwise velocity component of that mode for $L / D=1.2,1.5,2$ and 3 . When $L / D$ increases sufficiently the size of the perturbation stops being constrained by the upstream wall and it is more appropriate to use the characteristic length of the perturbation $L_{P} / D$ in the flow direction in the aforementioned exponential law, as seen in figure $8(a)$. That perturbation length can be defined, to use as reference, as the horizontal distance between the $50 \%$ of the value of the leftmost peak of the spanwise velocity component of the perturbation and the downstream wall of the cavity:

$$
R e_{\text {Drit }}=C_{1} \exp \left(C_{2} D / L_{P}\right) \text {. }
$$

The parameters $C_{1}$ and $C_{2}$ for the two lobes of each of the first and second modes can be found on table 3 (left-hand side). 




(b)
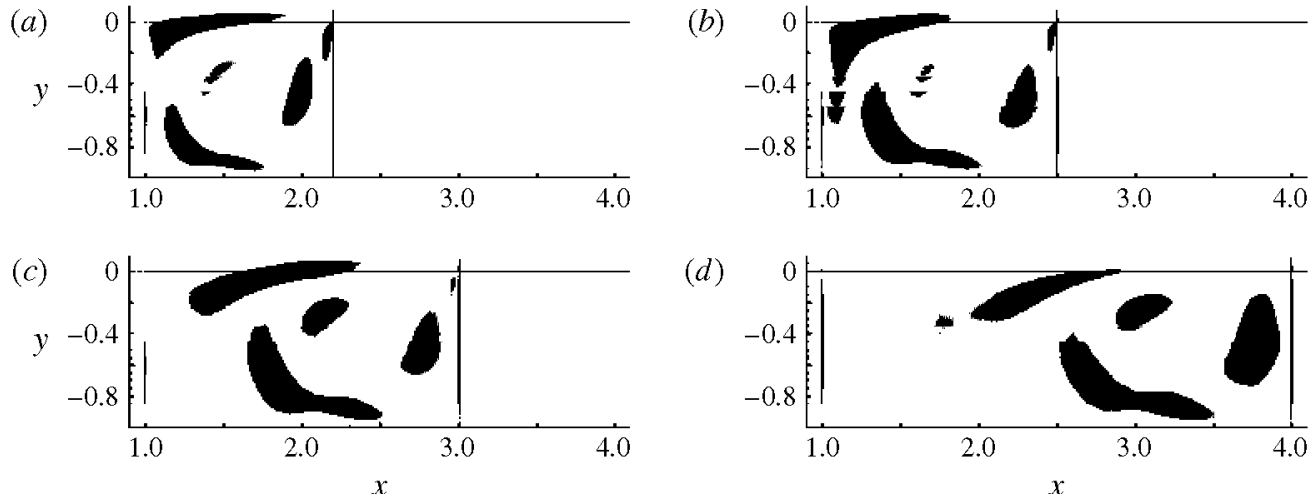

FIGURE 9. (Colour online) Qualitative representation of the spanwise velocity component of the low- $\beta$ lobe of Mode I: (a) $L / D=1.2$, (b) $L / D=1.5$, (c) $L / D=2$ and (d) $L / D=3$.

Mode

Mode I low- $\beta$ branch

Mode I high- $\beta$ branch

Mode II low- $\beta$ branch

Mode II high- $\beta$ branch

$\begin{array}{ccccc}C_{1} & C_{2} & \text { Mode } & K_{1} & K_{2} \\ 112.1 & 3.79 & \text { Mode II } & 0.0069 & 0.696 \\ 376.0 & 2.35 & \text { Mode I } & 0.0167 & 0.551 \\ 234.5 & 3.11 & \text { Mode III } & 0.0340 & 0.635 \\ 411.7 & 2.11 & \text { Stable mode } & 0.0545 & 0.609 \\ & & \text { Stable mode } & 0.0854 & 0.645\end{array}$

TABLE 3. The parameters $C_{1}$ and $C_{2}$ of (4.1) for the different modes (left) and the parameters $K_{1}$ and $K_{2}$ of (4.2) for the different modes (right).

Similar behaviour occurs in the case of the $\beta$ of maximum amplification. While the modes are in the range in which $L_{P} / D \simeq L / D$ there is a linear relation between $L / D$ and $L_{z}$ (that is, an hyperbolic relation with $\beta$ ), as it can be seen in figure $8(b)$. Outside that range, substituting the characteristic length by $L_{P} / D$ yields that the curve is being followed for Mode I. In the case of Mode II the behaviour is as expected for the low- $\beta$ branch, while not so for the other one. The results presented lead to the conclusion that a proportionality relationship exists between the depth-to-width ratio and the length of maximum amplification $2 \pi / \beta$, for the critical eigenmodes, so as $L / D$, and consequently $L_{P} / D$ increases, the length of the perturbation in the spanwise direction tends to increase in similar proportion.

Another observation is the apparent relation between the frequency of the eigenmodes, expressed as the dimensionless Strouhal number, $S t$, and the cavity aspect ratio $L / D$. For a given aspect ratio there is little variation of the Strouhal number of the modes with the Reynolds number or the incoming boundary-layer thickness, so each mode appears to be associated with a characteristic frequency. Figure 10 is a plot of the dimensionless frequency of the different eigenmodes versus the aspect ratio. The frequency of some stable eigenmodes is also considered. Using the results of the present analysis a correlation between the two parameters can be constructed, which follows a potential law:

$$
S t=K_{1}(L / D)^{K_{2}} .
$$

The parameters $K_{1}$ and $K_{2}$ for the different modes can be found in table 3 (right-hand side). Very few results can be found in the literature on these three-dimensional 


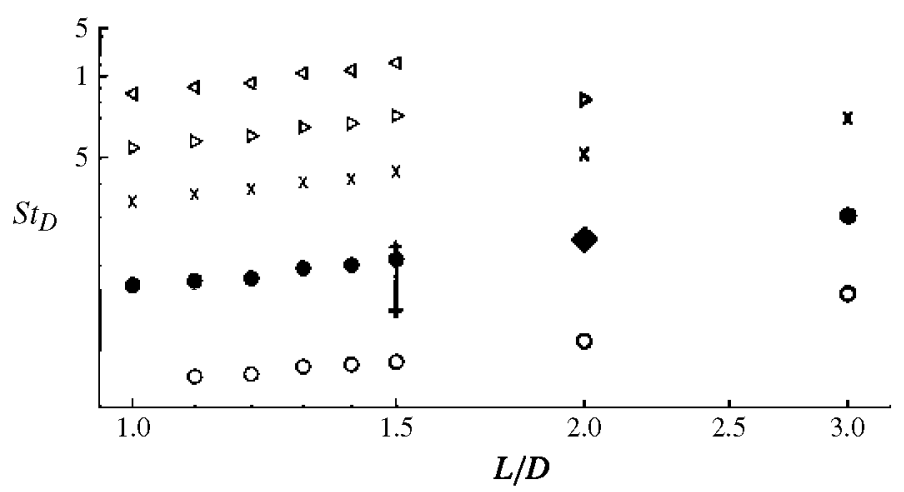

FIGURE 10. (Colour online) Strouhal number for the main oscillating modes versus the length-to-depth aspect ratio of the cavity, both in logarithmic scale. Symbols $\boldsymbol{\bullet}, \mathbf{0}, \times$ show the first, second and third unstable modes respectively. The other two $(\triangleleft, \triangleright)$, are stable modes for the range of parameters studied. The diamond (red online) shows the dominant frequency of Brès \& Colonius (2008), and the vertical line with crosses the range of frequencies obtained by Basley et al. (2013).

structures with low Strouhal numbers. However, the recent work of Basley et al. (2013) presents an experimental study on open cavity flow in which a broad band of frequencies corresponding to three-dimensional structures can be found. The range of those frequencies matches that found here, as do the results presented in Brès \& Colonius (2008).

\section{Summary}

The onset of three-dimensional instability of the incompressible flow over spanwise homogeneous flow in a rectangular cavity has been described. For a wide range of the parameters involved in the problem, the features of the dominant perturbations have been studied, allowing the extraction of relations between the different parameters and a description of the neutral hypersurface, through cuts of several planes of the parameter space. Two main three-dimensional modes appear in the majority of the cases studied: a bifurcated mode, with both a travelling and a stationary (and more unstable) lobe, as well as a purely travelling mode. The stability behaviour of those modes with respect to variations of all the parameters has been reported, allowing predictions of the stability of any configuration sufficiently close to critical conditions using only geometrical features of the cavity. In particular, the relations between the characteristic Strouhal number of the different eigenmodes, their critical Reynolds number and the $L / D$ ratio have been characterised. Such information should useful not only in order to predict instabilities of a given open cavity, but also as basis for reduced-order models of global flow instability and subsequent flow control, both theoretical and experimental.

\section{REFERENCES}

Albensoeder, S., Kuhlmann, H. C. \& Rath, H. J. 2001 Three-dimensional centrifugal-flow instabilities in the lid-driven-cavity problem. Phys. Fluids 13 (1), 121-135.

Alizard, F., Robinet, J. C. \& Gloerfelt, X. 2012 A domain decomposition matrix-free method for global linear stability. Comput. Fluids 66, 63-84. 


\section{On linear instability mechanisms in incompressible open cavity flow}

Barbagallo, A., Sipp, D. \& Schmid, P. J. 2009 Closed-loop control of an open cavity flow using reduced-order models. J. Fluid Mech. 641, 1-50.

Basley, J., PAstur, L. R., Delprat, N. \& Lusseyran, F. 2013 Space-time aspects of a threedimensional multi-modulated open cavity flow. Phys. Fluids 25, 064105.

Basley, J., Pastur, L. R., Lusseyran, F., Faure, T. M. \& Delprat, N. 2011 Experimental investigation of global structures in an incompressible cavity flow using time-resolved PIV. Exp. Fluids 50 (4), 905-918.

BRÈs, G. A. 2007 Numerical simulations of three-dimensional instabilities in cavity flows. PhD thesis, California Institute of Technology.

Brès, G. A. \& Colonius, T. 2008 Three-dimensional instabilities in compressible flow over open cavities. J. Fluid Mech. 599, 309-339.

Cattafesta III, L. N., Garg, S., Kegerise, M. S. \& Jones, G. S. 1998 Experiments on compressible flow-induced cavity oscillations. ALAA Paper 98-2912.

Colonius, T., Rowley, C. W. \& Theofilis, V. 2001 Global instabilities and reduced-order models of cavity flow oscillations. In First Symposium on Global Flow Instability and Control (ed. V. Theofilis, T. Colonius \& A. Seifert), 23-25 September 2001, Creta Maris, Hersonissos, Greece.

Faure, T. M., Pastur, L. R., Lusseyran, F., Fraigneau, Y. \& Bisch, D. 2009 Three-dimensional centrifugal instabilities development inside a parallelepipedic open cavity of various shape. Exp. Fluids 47 (3), 395-410.

GHARIB, M. \& RoshKo, A. 1987 The effect of flow oscillations on cavity drag. J. Fluid Mech. 177, 501-530.

Gómez, F. J. 2013 Matrix-free time-stepping methods for the solution of triglobal instability problems. $\mathrm{PhD}$ thesis, Universidad Politécnica de Madrid.

Gómez, F., Gómez, R. \& Theofilis, V. 2014 On three-dimensional global linear instability analysis of flows with standard aerodynamics codes. Aerosp. Sci. Technol. 32 (1), 223-234.

Gómez, F., Le Clainche, S., Paredes, P., Hermanns, M.\& Theofilis, V. 2012 Four decades of studying global linear instability: progress and challenges. AIAA J. 50, 2731-2743.

González, L. M., Ahmed, M., Kuhnen, J., Kuhlmann, H. C. \& Theofilis, V. 2011 Three-dimensional flow instability in a lid-driven isosceles triangular cavity. J. Fluid Mech. 675, 369-396.

Gresho, P. M. \& SANI, R. L. 1987 On pressure boundary conditions for the incompressible Navier-Stokes equations. Intl J. Numer. Meth. Fluids 7 (10), 1111-1145.

Kegerise, M. A., Spina, E. F., Garg, S. \& Cattafesta III, L. N. 2004 Mode-switching and nonlinear effects in compressible flow over a cavity. Phys. Fluids 16, 678-687.

Koseff, J. R. \& Street, R. L. 1984 On endwall effects in a lid-driven cavity flow. Trans. ASME: J. Fluids Engng 106, 385-389.

NeARY, M. D. \& StePhanoff, K. D. 1987 Shear-layer-driven transition in a rectangular cavity. Phys. Fluids 30 (10), 2936-2946.

Pereira, J. C. F. \& SousA, J. M. M. 1995 Experimental and numerical investigation of flow oscillations in a rectangular cavity. Trans. ASME: J. Fluids Engng 117 (1), 68-74.

Rockwell, D. 1977 Prediction of oscillation frequencies for unstable flow past cavities. Trans. ASME: J. Fluids Engng 99, 294-300.

RoCKWELL, D. \& KNISELY, C. 1980 Observations of the three-dimensional nature of unstable flow past a cavity. Phys. Fluids 23, 425-431.

Rockwell, D. \& KNisely, C. 1987 The organized nature of flow impingement upon a corner. J. Fluid Mech. 93, 413-432.

RocKWELl, D. \& NAUdASCHER, E. 1979 Self-sustained oscillations of impinging free shear layers. Annu. Rev. Fluid Mech. 11, 67-94.

Rossiter, J. E. 1964 Wind-tunnel experiments on the flow over rectangular cavities at subsonic and transonic speeds. Aeronaut. Res. Counc. R. \& M. 3438.

Rowley, C., Colonius, T. \& BASU, A. 2002 On self-sustained oscillations in two-dimensional compressible flow over rectangular cavities. J. Fluid Mech. 455, 315-346. 


\section{F. Meseguer-Garrido, J. de Vicente, E. Valero and V. Theofilis}

SANI, R. L. \& GRESHO, P. M. 1994 Résumé and remarks on the open boundary condition minisymposium. Intl J. Numer. Meth. Fluids 18 (10), 983-1008.

SAROHIA, V. 1975 Experimental and analytical investigation of oscillations in flows over cavities. $\mathrm{PhD}$ thesis, California Institute of Technology.

SIPP, D. 2012 Open-loop control of cavity oscillations with harmonic forcings. J. Fluid Mech. 708, 439-468.

Sipp, D. \& LeBedeV, A. 2007 Global stability of base and mean flows: a general approach and its applications to cylinder and open cavity flows. J. Fluid Mech. 593, 333-358.

Theofilis, V. 2000a Globally unstable basic flows in open cavities. Maui, HI: AIAA Paper 20001965.

Theofilis, V. $2000 \mathrm{~b}$ On steady-state flow solutions and their non-parallel global linear instability. In Eighth European Turbulence Conference (ed. C. Dopazo), 27-30 June 2000, Barcelona, Spain, pp. 35-38.

Theofilis, V. 2011 Global linear instability. Annu. Rev. Fluid Mech. 43, 319-352.

Theofilis, V. \& Colonius, T. 2003 An algorithm for the recovery of 2- and 3-d biglobal instabilities of compressible flow over 2-d open cavities. In AIAA Paper 2003-4143.

Theofilis, V. \& Colonius, T. 2004 Three-dimensional instablities of compressible flow over open cavities: direct solution of the biglobal eigenvalue problem. AIAA Paper 2006-2877.

DE VICENTE, J. 2010 Spectral multi-domain method for the global instability analysis of complex cavity flows. PhD thesis, Universidad Politécnica de Madrid.

de Vicente, J., Basley, J., Theofilis, V., Soria, J. \& Meseguer-Garrido, F. 2014 Threedimensional instabilities over a rectangular open cavity: from linear analysis to experimentation. J. Fluid Mech. 748, 189-220.

de Vicente, J., Valero, E., González, L. M. \& Theofilis, V. 2004 Spectral multi-domain methods for biglobal instability analysis of complex flows over open cavity configurations. AIAA Paper 2004-2544.

YAMOUNI, S., SIPP, D. \& JACQUIN, L. 2013 Interaction between feedback aeroacoustic and acoustic resonance mechanisms in a cavity flow: a global stability analysis. J. Fluid Mech. 717, 134-165. 